\title{
Analysis of Metabolic Changes in Plant Pathosystems by Imprint Imaging DESI-MS
}

\author{
Alessandra Tata, ${ }^{1,2}$ Consuelo J. Perez, ${ }^{1}$ Tanam S. Hamid, ${ }^{1}$ Mark A. Bayfield, ${ }^{3}$ \\ Demian R. Ifa ${ }^{1}$ \\ ${ }^{1}$ Centre for Research in Mass Spectrometry, Department of Chemistry, York University, Toronto, Ontario, Canada \\ ${ }^{2}$ ThoMSon Mass Spectrometry Laboratory, Institute of Chemistry, University of Campinas-UNICAMP, Campinas, Sao Paulo, \\ Brazil \\ ${ }^{3}$ Department of Biology, York University, Toronto, Ontario, Canada
}

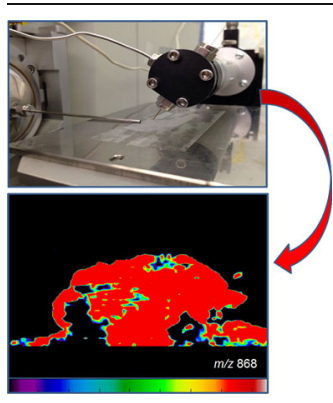

Abstract. The response of plants to microbial pathogens is based on the production of secondary metabolites. The complexity of plant-pathogen interactions makes their understanding a challenging task for metabolomic studies requiring powerful analytical approaches. In this paper, the ability of ambient mass spectrometry to provide a snapshot of plant metabolic response to pathogen invasion was tested. The fluctuations of glycoalkaloids present in sprouted potatoes infected by the phytopathogen Pythium ultimum were monitored by imprint imaging desorption electrospray ionization mass spectrometry (DESI-MS). After $8 \mathrm{~d}$ from the inoculation, a decrease of the relative abundance of potato glycoalkaloids $\alpha$-solanine $(\mathrm{m} / z \mathrm{706})$ and $\alpha$-chaconine $(\mathrm{m} / \mathrm{z}$ 722) was observed, whereas the relative intensity of solanidine $(\mathrm{m} / z$ 398), solasodenone $(\mathrm{m} / \mathrm{z} 412)$, solanaviol $(\mathrm{m} / \mathrm{z} 430)$, solasodiene $(\mathrm{m} / \mathrm{z} 396)$, solaspiralidine $(\mathrm{m} / \mathrm{z} 428)$, $\mathrm{\gamma}$-solanine/ $\mathrm{\gamma}$ chaconine $(\mathrm{m} / \mathrm{z} 560)$, $\beta$-solanine $(\mathrm{m} / \mathrm{z} 706)$, and $\beta$-chaconine $(\mathrm{m} / \mathrm{z} 722)$ increased. The progression of the disease, expressed by the development of brown necrotic lesions on the potato, led to the further decrease of all the glycoalkaloid metabolites. Therefore, the applicability of imprint imaging DESI-MS in studying the plant metabolic changes in a simple pathosystem was demonstrated with minimal sample preparation.

Keywords: Imaging mass spectrometry, Ambient ionization, Desorption electrospray ionization

Received: 29 August 2014/Revised: 27 October 2014/Accepted: 28 October 2014/Published Online: 16 December 2014

\section{Introduction}

$\mathrm{P}$ lants represent a rich source of nutrients for many organisms, including bacteria, fungi, protists, insects, and vertebrates [1]. The response of plants to microbial pathogens is based on the production of secondary metabolites. In fact, although lacking an immune system comparable to animals, plants have developed a stunning array of chemicals meant to face microbial attacks before extensive damage occurs [2]. Generally, studies on plant-pathogen interactions take interest in the biology of metabolism regulation; however, they can serve to provide strategies for the development and standardization of high-throughput metabolomics and for the

Electronic supplementary material The online version of this article (doi:10.1007/s13361-014-1039-0) contains supplementary material, which is available to authorized users.

Correspondence to: Demian Ifa; e-mail: ifadr@yorku.ca improvement of plant genetic engineering. The complexity of plant-pathogen interactions makes their understanding a challenging task requiring powerful analytical approaches. A few studies of plants pathosystems are available in literature, such as investigations of the metabolic response of tomato leaves [3], rice [4], and tobacco [5], upon infection with Pseudomonas syringae pv. tomato, Xanthomonas oryzae pv. oryzae, and Phythophthora parasitica, respectively. Investigations of the metabolomic changes of potato after infection with Phytophthora infestans were carried out by liquid chromatography mass spectrometry (LC-MS) [6].Fourier transform ion cyclotron resonance/mass spectrometry (FT-ICR/MS) was applied to the study of interactions in the potato sprout Rhizoctonia solani pathosystem and the fluctuations in the global metabolome of sprouts [7]. These analytical approaches may be laborious and time-consuming. Moreover, it is worth noting that the knowledge of precise functions of the secondary metabolites, produced in response to a pathogen attack, is often limited by insufficient characterization of their spatial 
distribution [8]. Therefore, here we report, for the first time, the application of imprint imaging desorption electrospray ionization mass spectrometry (DESI-MSI) to the study of the fluctuations of glycoalkaloids present in sprouted potatoes infected by the phytopathogen Pythium ultimum. The potato (Solanum tuberosum) is an important foodstuff in the world and P. ultimum is one of the most aggressive soil-borne fungal pathogens [9]. When performed under ambient conditions, sample pretreatment is minimal and sometimes unnecessary, thus simplifying the protocol while maintaining the high quality of the information that can be obtained. As already defined elsewhere [10], DESI is a spray-based ambient MSI technique that allows the direct sampling of surfaces in the open air. When used with a software-controlled moving stage, the sample is rastered underneath the DESI ionization probe, and through the time domain, $\mathrm{m} / \mathrm{z}$ information is correlated with the spatial distribution of chemical species [11]. Analysis of the spatial distribution of compounds in tissues by MSI is commonly performed using thin tissue sections obtained by cryosectioning bulk tissue in a cryostat and then thaw-mounted onto glass slides [12]. On the other hand, direct analysis of plant products, like leaves, has been reported by ambient MS [13, 14]. Alternatively, the use of blotting or imprinting techniques, in which the chemicals are initially transferred to flat, hard surfaces, is an approach that has been successfully applied in several imaging MS techniques [15-19]. Imprinting is a simple technique that allows MS imaging analysis of non-flat or irregular surfaces without the need to use a cryostat. Recently, imprints of potato sprout (Solanum tuberosum L.), gingko leaves (Gingko biloba L.), and strawberries (Fragaria x ananassa Duch), assisted by high temperature and/or solvent extraction, were successfully imaged by DESI-MS [20]. Hemalatha et al. described the chemical differences of leaves and flowers of Madagascar periwinkle by thin layer chromatography (TLC) imprint DESI-MS [21].The application of imprint imaging DESI-MS to the study of the fluctuations of glycoalkaloids of sprouted potatoes infected by the phytopathogen P. ultimum is reported in this manuscript. The bioanalytical method developed provides a fast and direct snapshot of the sprout's metabolic changes and perturbations that result from pathogen invasion with no need for time-consuming extractions. To the best of our knowledge, this is the first metabolic study of plant pathosystems by imaging ambient mass spectrometry.

\section{Experimental}

\section{Materials}

Methanol $(\mathrm{MeOH})$ and acetonitrile $(\mathrm{ACN})$ were purchased from Sigma Aldrich (Oakville, ON, Canada).The transparent tape (3M Highland, 5910) was bought at the York University bookstore and the potatoes at the local supermarket (Metro Richelieu Inc., Toronto, ON, Canada). The phytopathogen Pythium ultimum (ATCC 56081) was bought from Cederlane (Burlington, ON, Canada) and the potato dextrose broth (PDB) was also obtained from Sigma Aldrich (Oakville, ON, Canada).

\section{Inoculation of Potatoes}

Pre-sprouting potato tubers were kept in the dark at room temperature for $2 \mathrm{wk}$.

Tubers were washed under running tap water and air dried. Then they were sliced with a sterile knife and the surface of the slices was sterilized in $1 \%$ bleach for $15 \mathrm{~s}$, rinsed three times in sterile distilled water and then rapidly flamed. The slices were inoculated with P. ultimum previously grown in PDB for $8 \mathrm{~d}$ at $24^{\circ} \mathrm{C}$ and 200 revolutions per min (rpm). The infected potatoes were analyzed by DESI-MS and imaging DESI-MS at room temperature in natural light after $8 \mathrm{~d}$ of incubation.

\section{DESI-MS and DESI-MSI Experiments}

Different control $(n=3)$, infected $(n=3$ at $8 \mathrm{~d}$ from the inoculation of P. ultimum), and damaged ( $n=3$, at $21 \mathrm{~d}$ from the inoculation of $P$. ultimum) potatoes were sectioned using a knife and manually imprinted by pressing the tape onto them for $5 \mathrm{~s}$. Then, the imprinted tapes were placed in a lab-built 2D moving stage (described elsewhere [22]) using double-sided tape and analyzed by DESI-MS in the positive ion mode. All MS experiments were performed using a Thermo Fisher Scientific LTQ mass spectrometer (San Jose, CA, USA). Data were acquired and processed using Xcalibur 2.0 software (Thermo Fisher Scientific). Typical instrumental parameters used were $4.5 \mathrm{kV}$ capillary voltage and $275^{\circ} \mathrm{C}$ capillary temperature. A MeOH:ACN (1:1) solution was used as spray solvent and delivered at the flow rate of $3 \mu \mathrm{L} \mathrm{min}{ }^{-1}$. Mass spectra were acquired as full scans, in the positive ion mode, over the mass range from $\mathrm{m} / \mathrm{z} 200$ to 1000 . The sprayer-to-surface distance was $1.0-1.5 \mathrm{~mm}$, the sprayer to inlet distance was $4-8 \mathrm{~mm}$, an incident spray was set at $52^{\circ}$, and a collection angle of $10^{\circ}$ was used. The identification of the analyte ions was confirmed by tandem mass spectrometry (MS/MS) using collision-induced dissociation with collision energy of $25 \%-35 \%$ (manufacturer's unit). In order to acquire DESIMS images from the control and infected samples, the imprints were scanned in horizontal rows separated by 150 to $200 \mu \mathrm{m}$ vertical steps until the entire sample was analyzed. The lines were scanned at a constant velocity in the range of 414 to $714 \mathrm{~m} / \mathrm{s}$ and the scan time was set in the range from 0.43 to $0.56 \mathrm{~s}$. A lateral spatial resolution (pixel size) in the range of 150 to $200 \mu \mathrm{m}$ could be achieved under these conditions.

\section{Data Processing}

The MS spectra were processed by QualBrowserXcalibur. ImageCreator ver. 3.0 software was used to convert the Xcalibur 2.0 mass spectra files (.raw) into a format compatible with BioMap (freeware, http://www.maldi-msi.org/), which was used to process the mass spectral data and to generate $2 \mathrm{D}$ spatially resolved ion images. The identification of the metabolites was achieved by tandem mass spectrometry (MS/MS) and a literature search. 

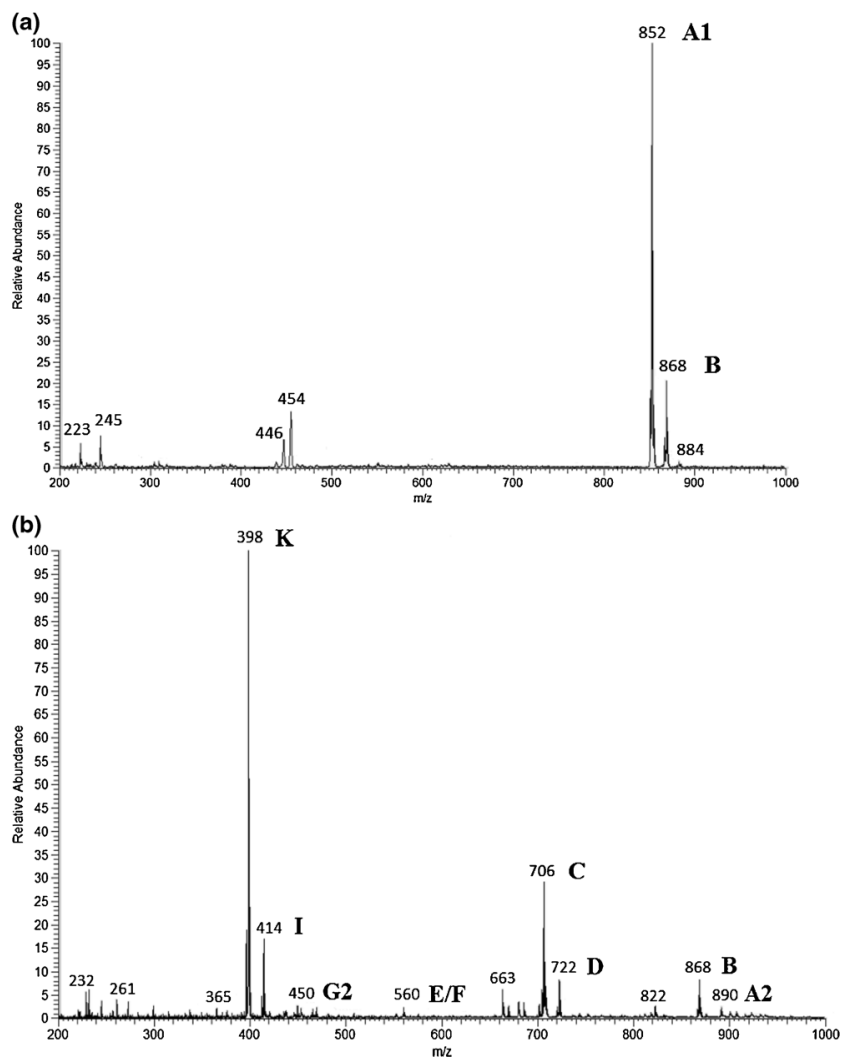

Figure 1. Full scan DESI-MS of (a) control potato and (b) $8 \mathrm{~d}$ infected potato. The changes in the glycoalkaloid metabolism can be observed with the time. Assignments of the metabolites are reported in Table 1

\section{Results and Discussion}

A DESI-MS profile acquired for an imprinted control potato at the sprout is shown in Figure 1a. The mass spectrum is mainly characterized by the ions of $\mathrm{m} / \mathrm{z} 852$ and 868 corresponding to the glycoalkaloids $\alpha$-chaconine and $\alpha$-solanine. These are the most abundant potato toxins and they usually comprise more than $95 \%$ of the total steroidal glycoalkaloid content [23]. Both toxins act as a defensive barrier for protection against pathogens [24]. DESI-MSI of imprinted sprout was also performed. The DESI-MSI of the control potato is reported in Supplementary Figure $1 \mathrm{~S}$. Well-defined images of the ions of $\mathrm{m} / \mathrm{z} 852$ and 868 can be obtained by regular imprinting on tape. Owing to the high water content of the sample, no solvent or thermal assistance are needed to get highly detailed images. A reproducible heterogeneous distribution of the ions of $\mathrm{m} / \mathrm{z} 852$ and 868 can be observed in the sprout. In accordance with the results reported by Friedman et al. in 1992 using highperformance liquid chromatography (HPLC) [25] and $\mathrm{Ha}$ et al. in 2012 by matrix assisted laser desorption ionization (MALDI)-IMS [26], the distribution of the $\alpha$-chaconine and $\alpha$ solanine greatly varies when comparing the sprout and the tuber. As the total glycoalkaloid content was reported to range (in $\mathrm{mg}$ per $100 \mathrm{~g}$ of fresh weight) from 2 for tuber to 997 for sprout, a similar variance in distribution (see ion of $\mathrm{m} / \mathrm{z} 868$ ) can be observed in Supplementary Figure S1 using imaging DESI-MS. These findings have implications for food safety and plant breeding since $\alpha$-chaconine is more embryotoxic and has a greater ability to induce liver enzymes than $\alpha$-solanine $[27,28]$.

In the infected potato ( $8 \mathrm{~d}$ after inoculation) the ions mentioned above were much lower in their relative abundance. The protonated forms of the glycoalkaloids solasodiene $(\mathrm{m} / \mathrm{z} 396)$, aglycon solanidine $(\mathrm{m} / \mathrm{z} 398)$, solasodenone $(\mathrm{m} / \mathrm{z} 412)$, solasodine $(\mathrm{m} / \mathrm{z} 414)$, solanaviol $(\mathrm{m} / \mathrm{z} 430)$, solaspiralide $(\mathrm{m} / \mathrm{z}$ 428), $\gamma$-solanine $/ \gamma$-chaconine $(\mathrm{m} / z$ 560), $\beta$-solanine $(\mathrm{m} / z$ 706), and $\beta$-chaconine $(\mathrm{m} / \mathrm{z}$ 722) can be seen in Figure $1 \mathrm{~b}$. Table 1 reports the assigned metabolites, their molecular formula, their monoisotopic mass and the observed $\mathrm{m} / \mathrm{z}$, and the type of ion. Figure 2 reports the structures of the observed metabolites. All ions were fully assigned by DESI-MS/MS and a literature search $[6,7]$. The MS/MS experiments were performed directly on the imprinted material; the MS/MS spectra are reported in the Supplementary Information. The MS fragmentations of $\alpha$ chaconine, $\alpha$-solanine, $\beta$-solanine, $\beta$-chaconine, and $\gamma$ -

Table 1. Characteristic Ions Observed in the Control and the Infected Sprouted Potatoes in Positive Ion Mode

\begin{tabular}{|c|c|c|c|c|c|c|}
\hline Origin & Metabolite reference & Metabolite $^{\mathrm{a}}$ & Molecular formula & Monoisotopic mass (Da) & Observed $\mathrm{m} / \mathrm{z}$ & Assignments \\
\hline \multirow[t]{14}{*}{ Steroidal alkaloids of potato } & A1 & \multirow[t]{2}{*}{$\alpha$-Chaconine } & \multirow[t]{2}{*}{$\mathrm{C}_{45} \mathrm{H}_{73} \mathrm{O}_{14} \mathrm{~N}$} & \multirow[t]{2}{*}{851.5031} & 852 & {$[\mathrm{M}+\mathrm{H}]^{+}$} \\
\hline & A2 & & & & 890 & {$[\mathrm{M}+\mathrm{K}]^{+}$} \\
\hline & B & $\alpha$-Solanine & $\mathrm{C}_{45} \mathrm{H}_{73} \mathrm{O}_{15} \mathrm{~N}$ & 867.4980 & 868 & {$[\mathrm{M}+\mathrm{H}]^{+}$} \\
\hline & $\mathrm{C}$ & $\beta$-Chaconine & $\mathrm{C}_{39} \mathrm{H}_{63} \mathrm{O}_{10} \mathrm{~N}$ & 705.4452 & 706 & {$[\mathrm{M}+\mathrm{H}]^{+}$} \\
\hline & $\mathrm{D}$ & $\beta$-Solanine & $\mathrm{C}_{39} \mathrm{H}_{63} \mathrm{O}_{11} \mathrm{~N}$ & 721.4401 & 722 & {$[\mathrm{M}+\mathrm{H}]^{+}$} \\
\hline & $\mathrm{E}$ & $\gamma$-Chaconine & $\mathrm{C}_{33} \mathrm{H}_{53} \mathrm{O}_{6} \mathrm{~N}$ & 559.3873 & 560 & {$[\mathrm{M}+\mathrm{H}]^{+}$} \\
\hline & $\mathrm{F}$ & $\gamma$-Solanine & \multirow{3}{*}{$\mathrm{C}_{27} \mathrm{H}_{41} \mathrm{NO}_{3}$} & \multirow{3}{*}{427.3086} & & \\
\hline & G1 & \multirow[t]{2}{*}{ Solaspiralidine } & & & 428 & {$[\mathrm{M}+\mathrm{H}]^{+}$} \\
\hline & G2 & & & & 450 & {$[\mathrm{M}+\mathrm{Na}]^{+}$} \\
\hline & $\mathrm{H}$ & Solanaviol & $\mathrm{C}_{27} \mathrm{H}_{43} \mathrm{O}_{3} \mathrm{~N}$ & 429.3243 & 430 & {$[\mathrm{M}+\mathrm{H}]^{+}$} \\
\hline & I & Solasodine & $\mathrm{C}_{27} \mathrm{H}_{43} \mathrm{O}_{2} \mathrm{~N}$ & 413.3294 & 414 & {$[\mathrm{M}+\mathrm{H}]^{+}$} \\
\hline & $\mathrm{J}$ & Solasodenone & $\mathrm{C}_{27} \mathrm{H}_{41} \mathrm{O}_{2} \mathrm{~N}$ & 411.3137 & 412 & {$[\mathrm{M}+\mathrm{H}]^{+}$} \\
\hline & K & Solanidine & $\mathrm{C}_{27} \mathrm{H}_{43} \mathrm{NO}$ & 397.3345 & 398 & {$[\mathrm{M}+\mathrm{H}]^{+}$} \\
\hline & $\mathrm{L}$ & Solasodiene & $\mathrm{C}_{27} \mathrm{H}_{41} \mathrm{NO}$ & 395.3188 & 396 & {$[\mathrm{M}+\mathrm{H}]^{+}$} \\
\hline
\end{tabular}

aAliferis et al. 2012 [7]; Pushpa et al. 2014 [6] 
(a)

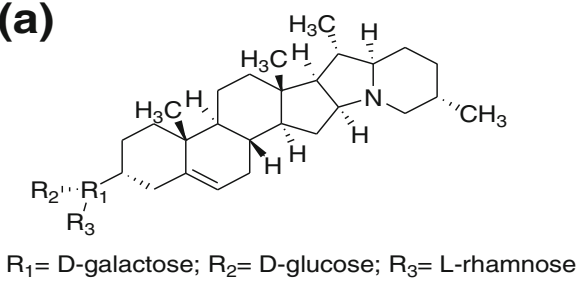

(c)

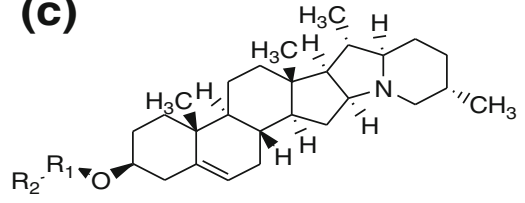
$\mathrm{R}_{1}=\mathrm{D}$-galactose; $\mathrm{R}_{2}=\mathrm{L}$-rhamnose

(e)

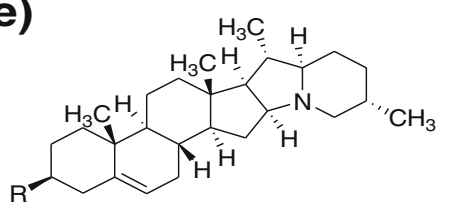
$\mathrm{R}=\mathrm{D}-$ galactose

(g)

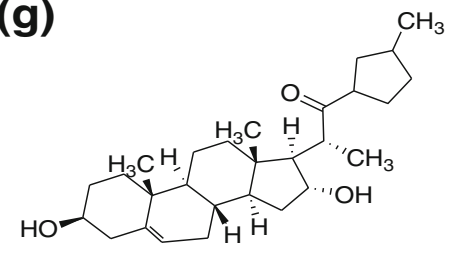

(i)<smiles>CC1CCC2(CC1)O[C@H]1C[C@H]3[C@@H]4CC=C5C[C@@H](O)CC[C@]5(C)C4CC[C@H]3[C@H]1[C@H]2C</smiles>

(k)

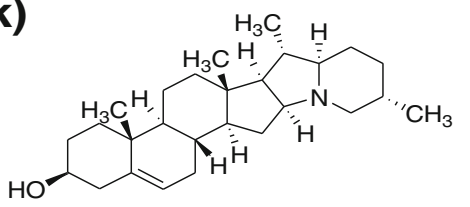

(b)

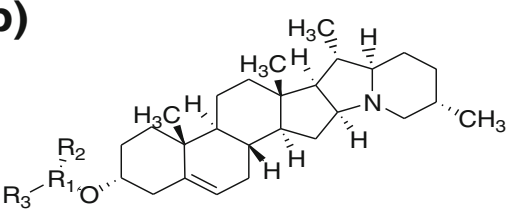
$R_{1}=D$-galactose $; R_{2}=D$-galactose $; R_{3}=L$-rhamnose

(d)

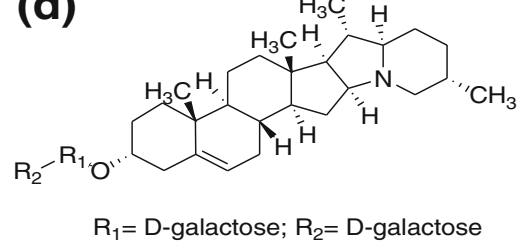

(f)

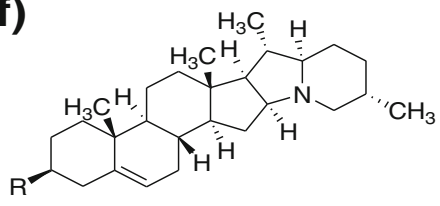

$R=$ D-galactose

(h)

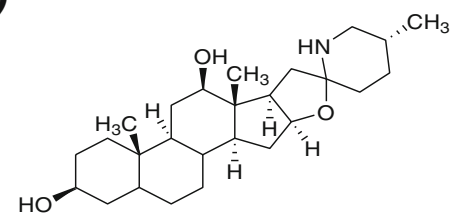

(j)

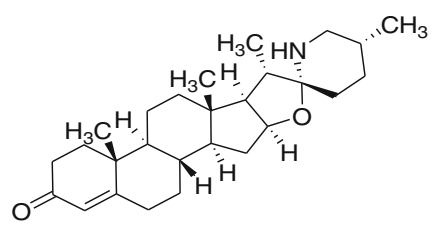

(I)<smiles>C[C@H]1CC[C@H]2O[C@H]3C[C@H]4C=CC5=CCCC[C@]5(C)[C@H]4CC[C@]3(C)[C@H]2[C@H]1C</smiles>

Figure 2. Structures of the glycoalkaloids observed in this study: (a) $\alpha$-Chaconine; (b) $\alpha$-Solanine; (c) $\beta$-Chaconine; (d) $\beta$-Solanine; (e) Y-Chaconine; (f) Y-Solanine; (g) Solaspiralidine; (h) Solanaviol; (i) Solasodine; (j) Solasodenone; (k) Solanidine; (l) Solasodiene

solanine $/ \gamma$-chaconine show the sequence of cleavages of the different sugar moieties (Supplementary Figures S2, S3, S10$\mathrm{S} 12)$. The MS-fragmentations of solasodiene, solanidine, solasodenone, solasodine, solanaviol, and solaspiralide are characterized by the cleavage of the six ring systems (Supplementary Figures S4-S9). These results are in agreement with the observations reported by Cahill et al.,who fully elucidated the mass fragmentation pathways of potato glycoalkaloids [29].

The most intense peak of the infected potato profile is the ion of $\mathrm{m} / \mathrm{z} 398$ corresponding to the aglycon solanidine. This observation perfectly matches with the literature $[30,31]$. In fact, it is known that fungal infection of potato sprouts activates biosynthetic pathways that lead to biosynthesis of alkaloids with a substantial increase in the aglycons solanidine and solasodine. This results in the de novo synthesis of several steroidal alkaloids that have solasodine or solanidine as their common aglycon. As reported in Scheme 1, the increased production of solasodine leads to the bioformation of the glycoalkaloids solasodenone $(\mathrm{m} / z 412)$, solanaviol $(\mathrm{m} / z 430)$, solasodiene ( $\mathrm{m} / \mathrm{z} 396)$, solasonine (not observed in our spectra), 

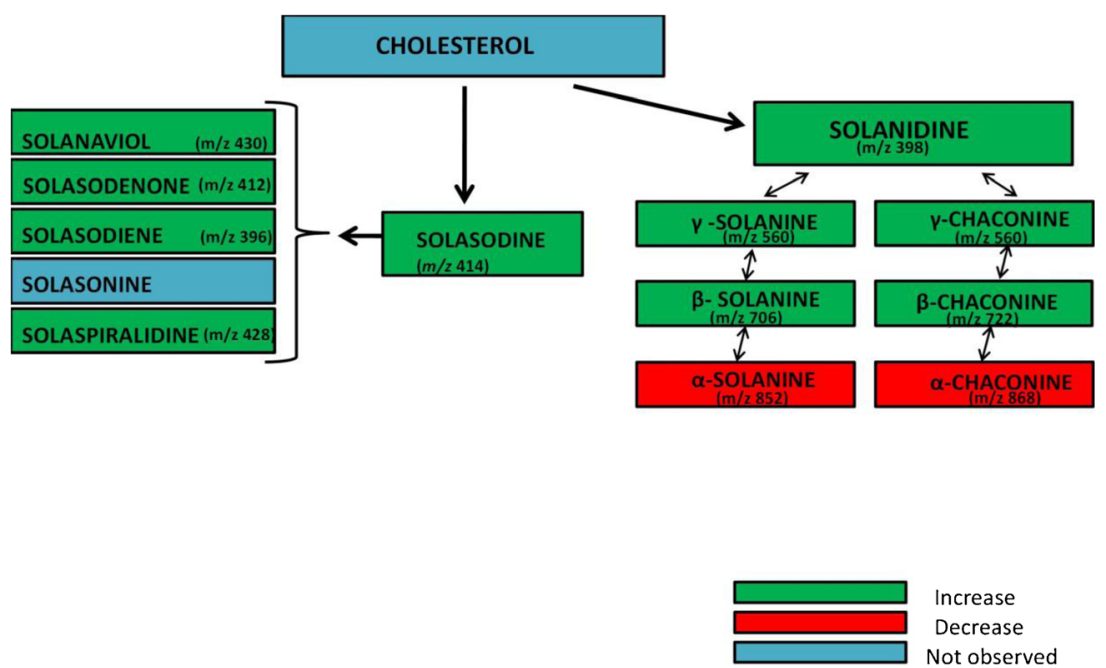

Scheme 1. Fluctuations in the potato sprout metabolic pathway leading to the biosynthesis of glycoalkaloids $8 \mathrm{~d}$ after infection by P. ultimum. The changes in metabolic expression are coded using colors as explained in the legend. Adapted from Aliferis et al. 2007 [7]

and solaspiralidine $(\mathrm{m} / \mathrm{z} 428)$, whereas the aglycon solanidine forms the $\gamma$-solanine $/ \gamma$-chaconine $(\mathrm{m} / \mathrm{z} 560), \beta$-solanine $(\mathrm{m} / z$ 706), and $\beta$-chaconine $(\mathrm{m} / \mathrm{z}$ 722).

Note that a strong decrease in the relative abundance of the main potato toxins $\alpha$-chaconine and $\alpha$-solanine was observed. This observation can be explained considering that successful pathogens avoid such toxicity by removing sugar chains from the molecule of glycoalkaloids (i.e., hydrolysis) by $\mathrm{pH}$ alteration [32]. The hydrolytic degradation of $\alpha$-solanine and $\alpha$ chaconine to their catabolic and less toxic $\beta$ - and $\gamma$-forms of $\mathrm{m} / \mathrm{z}$ 706 and 722, respectively, during sprout colonization by the phytopathogen Rhizoctonia solani has been reported recently by Aliferis et al. [7].

It seems that this process is a key contributor to the ability of phytopathogen to overcome the toxicity the glycoalkaloids [33]. Although not yet assessed in P. ultimum, it has been also demonstrated that the enzymes rhamnosidase and $\alpha$ chaconinase, purified from potato fungal pathogens, were able to convert $\alpha$-chaconine to $\beta$-chaconine via a stepwise removal of a sugar unit from the trisaccharide chain, rendering the metabolite less bioactive [34].

Investigating the spatial distribution of the plant secondary metabolites of infection and their different accumulation, in the vicinity of the site, allows finding the localization of distinct metabolic pathways [8]. Figure 3 shows the local distribution of the glycoalkaloids produced in response to the pathogen invasion by imaging DESI-MS. The glycoalkaloids are distributed in different localizations of the sprout. Despite an accurate literature search, unfortunately it is hard to explain their varying localizations inside the sprout from an anatomical point of view. In order to make visible the ions of $\mathrm{m} / \mathrm{z} 428$, 430,560 , and 852 , which have very low relative intensities, the color bar scale applied to all ions was normalized to a value below the maximum intensity of the base peak $(\mathrm{m} / \mathrm{z} 398)$. Therefore, the relative abundance of the other ions seems higher than the spectrum would suggest. Comparing Figure 4 and Supplementary Figure S1, change in accumulation of the glycolakaloids $\alpha$-solanine (it almost disappears) and the appearance the new metabolites biosynthesized after fungus invasion can be noticed. This observation, in accordance with the data reported by Kuc in 1984 showing the ability of tuber and sprout to accumulate new glycoalkaloids underneath the peel of sprout and tuber, immediately around the site of injury [35].

Three weeks after the inoculation, the potato sprout was imprinted again and new changes in the glycoalkaloid metabolism were observed. The DESI-MS spectrum reported in Figure 4 shows a decrease of the relative abundance of the ions of $\mathrm{m} / \mathrm{z} 396,412,414,706$, and 722, corresponding to the metabolites solasodiene, solasodenone, solasodine, $\beta$ - and $\gamma$-chaconine, and solanine, respectively. The progression of the disease, as it is expressed by the development of brown necrotic lesions, led to the further decrease of the glycoalkaloid metabolites. This result may prove that $P$. ultimum is tolerant to the glycoalkaloids, as previously shown in other glycoalkaloids containing plants attacked by phytopathogens [7]. In this study, we have demonstrated the suitability of DESI-MS for analyzing, in a qualitative way, the time dependence of metabolic changes of the potato after phytopathogen invasion. The results are in accordance with those obtained previously by Aliferis [7] and Pushpa [6] by conventional mass spectrometric techniques. The imprinting procedure on regular tape, with no need of thermo and/or solvent assistance, was demonstrated to be efficient and simple. It allowed the imaging analysis of non-flat, irregular surfaces without the use of a cryostat.

In this specific case, an important factor that facilitates the transfer of compounds, especially polar compounds, to the tape is the water content in the sample. This resulted in a successful imprint that enabled the acquisition of high-quality spectra and well-defined images. 

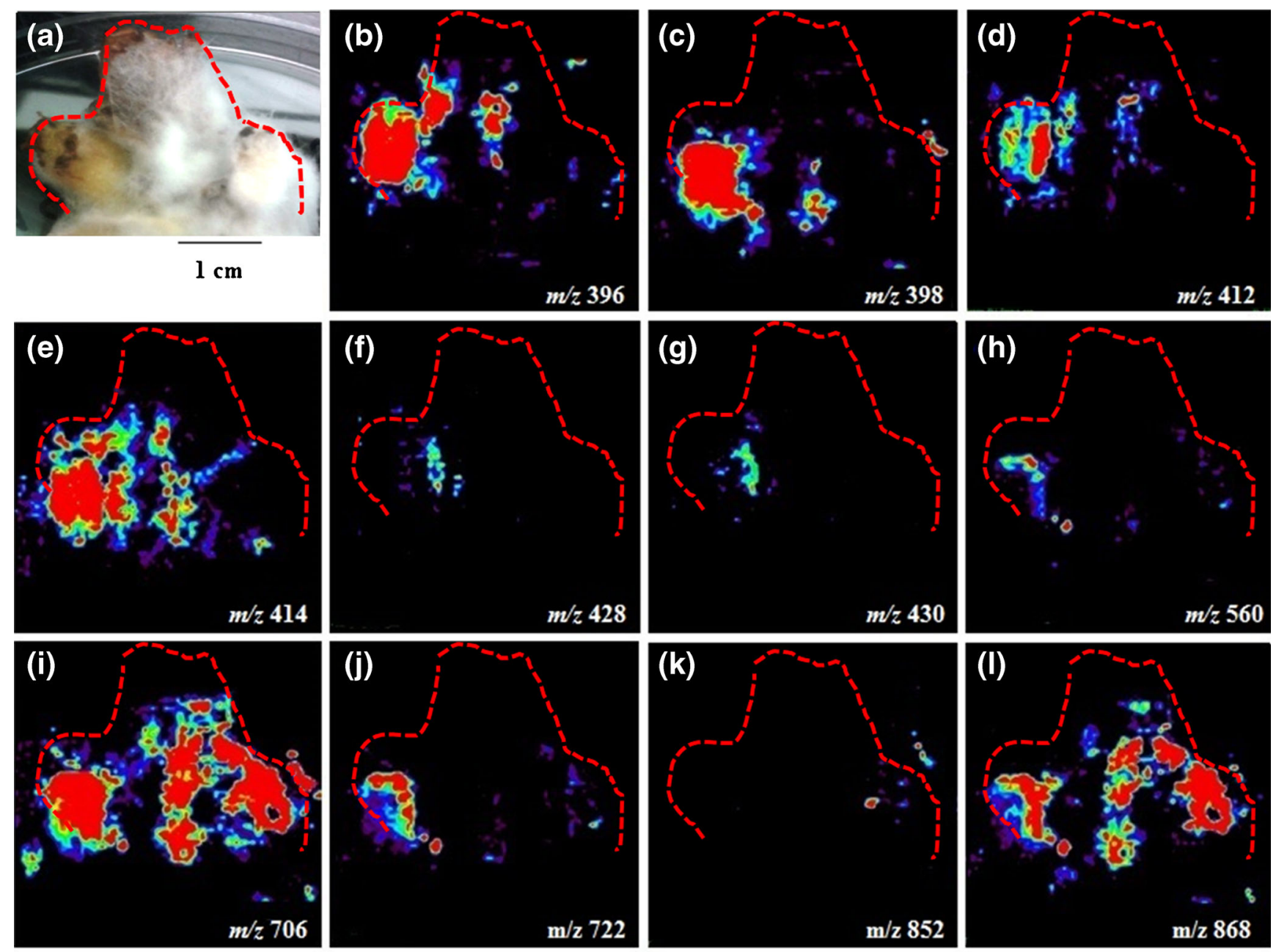

$0 \%$

$100 \%$

Figure 3. Imaging DESI-MS of an infected potato sprout imprinted on tape, $8 \mathrm{~d}$ after inoculation. Localization of the new metabolites biosynthesized after fungus invasion can be observed. The infected potato sprout is imprinted directly on tape without further manipulation. The red outline indicates the portion of the sprout and the tuber imaged

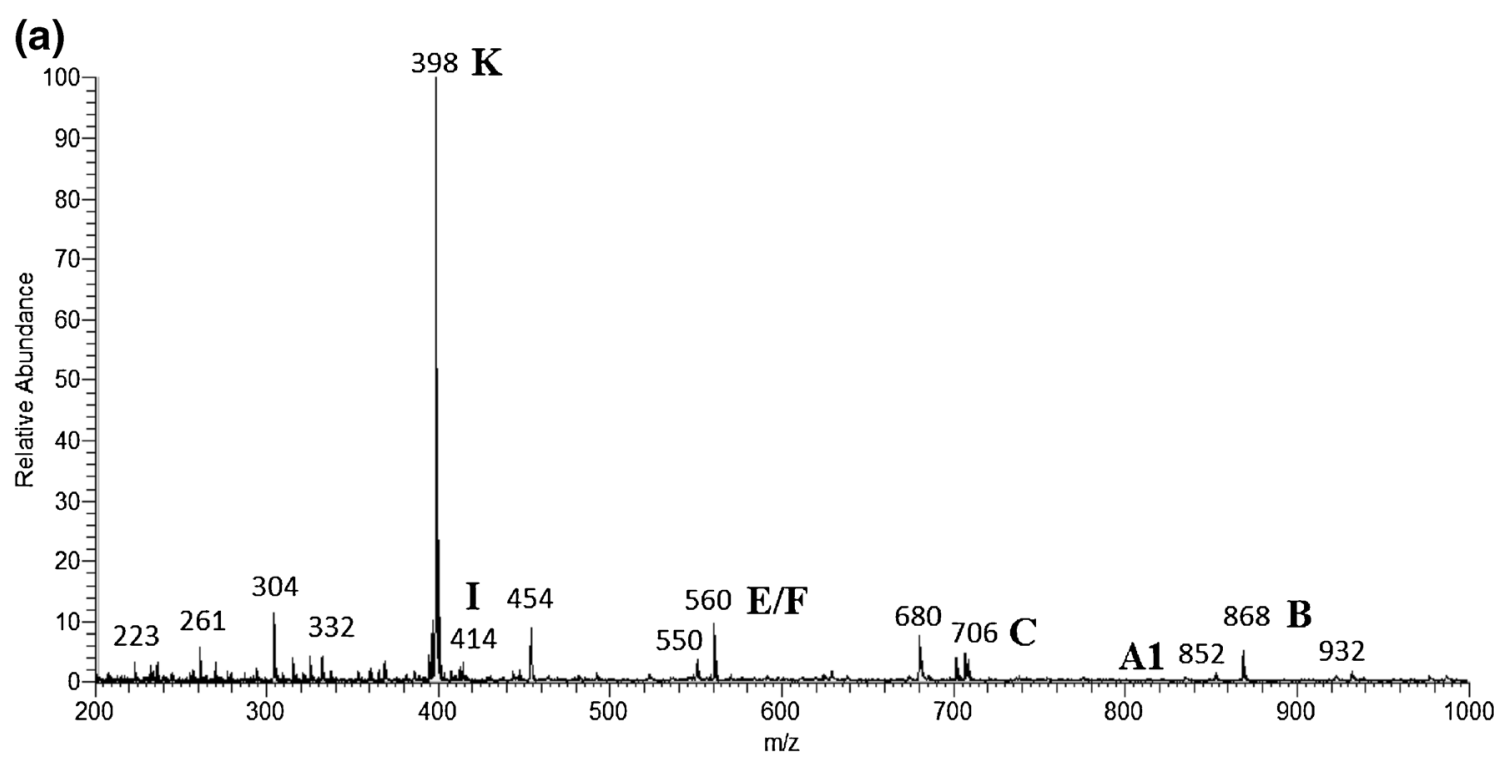

Figure 4. Blotting DESI-MS of damaged potato after $3 \mathrm{wk}$ from the inoculation 


\section{Conclusion}

The efficiency of DESI-MS in studying the plant metabolic changes in a simple pathosystem was demonstrated. The imprint on regular tape, coupled to DESI, allowed the MS profile and imaging of a complex system in a fast way, using minimal amount of sample with no need of laborious extractions. To the best of our knowledge, this is the first qualitative study of the plant chemical defense against a phytopathogen invasion by ambient mass spectrometry. Quantification of the glycotoxins and metabolites would be valuable in studies of plant pathosystems, especially for the determination of kinetic parameters. Quantitation is an important emerging topic that needs more attention in ambient ionization imaging mass spectrometry [10]. For the determination of analytes in intact tissues using imaging DESI-MS, previous efforts have focused on relative quantification [36]. However, absolute quantitation can also be done [37]. These experiments require the careful selection of internal standards and their homogeneous transfer to the sample. Calibrants and quality control samples should be used for the validation of the method and, in some cases, crossvalidation with other analytical techniques can still be necessary. Although quantitative experiments are promising, their robustness and real applicability to imaging mass spectrometry need to be further investigated [38].

\section{Acknowledgments}

The authors are grateful to Irina Oganyesian for technical support. The authors thank the Natural Science and Engineering Research Council of Canada (NSERC) and the Brazilian Science Foundation FAPESP (2013/15575-9) for financial assistance.

\section{References}

1. Freeman, B.C., Beattie, G.A.: The plant health instructor. Am. Phytopathol. Soc. St. Paul. (2008). doi:10.1094/PHI-I-2008-0226-01

2. Gunatilaka, L.A.: Natural products from plant-associated microorganisms: distribution, structural diversity, bioactivity, and implications of their occurrence. J. Nat. Prod. 69, 509-526 (2006)

3. López-Gresa, M.P., Maltese, F., Bellés, J.M., Conejero, V., Kim, H.K.: Metabolic response of tomato leaves upon different plant-pathogen interactions. Phytochem. Anal. 21, 89-94 (2010)

4. Sana, T.R., Fischer, S., Wohlgemuth, G., Katrekar, A., Jung, K.: Metabolomic and transcriptomic analysis of the rice response to the bacterial blight pathogen Xanthomonasoryzae pv oryzae. Metabolomics 6, 451465 (2010)

5. Cho, K., Kim, Y., Wi, S.J., Seo, J.B., Kwon, J., Chung, J.H., Park, K.Y., Nam, M.H.: Nontargeted metabolite profiling in compatible pathogeninoculated tobacco (Nicotiana tabacum L. cv. Wisconsin 38) using UPLC-Q-TOF/MS. J. Agric. Food Chem. 60(44), 11015-11028 (2012)

6. Pushpa, D., Yogendra, K.N., Gunnaiah, R., Kushalappa, A.C., Murphey, A.: Identification of late blight resistance related metabolites and genes in potato through nontargeted metabolomics. Plant Mol. Biol. Rep. 32, 584 595 (2014)

7. Aliferis, K.A.: FT-ICR/MS and GC-EI/MS metabolomics networking unravels global potato sprout's responses to Rhizoctonia solani infection. PLoS One 7, e42576 (2012)

8. Simon, C., Langlois-Meurinne, L., Bellvert Garmier, M., Didierlaurent, L., Massoud, K., Chaouch, S., Marie, A., Bodo, B., Kauffmann, S., Noctor, G., Saindrenan, G.: The differential spatial distribution of secondary metabolites in Arabidopsis leaves reacting hypersensitively to Pseudomonas syringae pv. tomato is dependent on the oxidative burst. J. Exp. Bot. 61(12), 3355-33570 (2010)

9. Whipps, J.M., Lumsden, R.D.: Biological control of Pythium species. BioSci. Technol. 1, 75-90 (1991)

10. Wu, C., Dill, A.L., Eberlin, L.S., Cooks, R.G., Ifa, D.R.: Mass spectrometry imaging under ambient conditions. Mass Spectrom. Rev. 32, 218-243 (2013)

11. Monge, M.E., Harris, G.A., Dwivedi, P., Fernández, F.M.: Mass spectrometry: recent advances in direct open air surface sampling/ionization. Chem. Rev. 113, 2269-2308 (2013)

12. Wu, C., Ifa, D.R., Manicke, N.E., Cooks, R.G.: Rapid, direct analysis of cholesterol by charge labeling in reactive desorption electrospray ionization. Analyst 135, 28-32 (2010)

13. Jackson, A.U., Tata, A., Wu, C., Perry, R.H., Haas, G., West, L., Cooks, R.G.: Direct analysis of Stevia leaves for diterpene glycosides by desorption electrospray ionization mass spectrometry. Analyst 134, 867-874 (2009)

14. Nemes, P., Vertes, A.: Laser ablation electrospray ionization for atmospheric pressure, in vivo, and imaging mass spectrometry. Anal. Chem. 79, 8098-8106 (2007)

15. Ifa, D.R., Srimany, A., Eberlin, L.S., Naik, H.R., Bhat, V., Cooks, R.G., Pradeep, T.: Tissue imprint imaging by desorption electrospray ionization mass spectrometry. Anal. Methods 3, 1910-1912 (2011)

16. Tata, A., Fernandes, A.M., Santos, V.G., Alberici, R.M., Araldi, D., Parada, C.A., Braguini, W., Veronez, L., Bisson, G.S., Reis, F.H., Alberici, L.C., Eberlin, M.N.: Nano-assisted laser desorption-ionization-MS imaging of tumors. Anal. Chem. 84, 6341-6345 (2012)

17. Tata, A., Montemurro, C., Porcari, M.A., Silva, K.C., Lopes de Faria, J.B., Eberlin, M.N.: Spatial distribution of theobromine - a low MW drug - in tissues via matrix-free NALDI-MS imaging. Drug Test Anal. 6, 949-952 (2014)

18. Caprioli, R.M., Farmer, T.B., Gile, J.: Molecular imaging of biological samples: localization of peptides and proteins using MALDI-TOF MS. Anal. Chem. 69, 4751-4760 (1997)

19. Masumori, N., Thomas, T.Z., Chaurand, P., Case, T., Paul, M., Kasper, S., Caprioli, R.M., Tsukamoto, T., Shappell, S.B., Matusik, R.J.: A probasinlarge $\mathrm{T}$ antigen transgenic mouse line develops prostate adenocarcinoma and neuroendocrine carcinoma with metastatic potential. Cancer Res. 61, 2239-2249 (2001)

20. Cabral, C.E., Mirabelli, M.F., Perez, C.J., Ifa, D.R.: Blotting assisted by heating and solvent extraction for DESI-MS Imaging. J. Am. Soc. Mass Spectrom. 24, 956-965 (2013)

21. Hemalatha, R.G., Pradeep, T.: Understanding the molecular signatures in leaves and flowers by desorption electrospray ionization mass spectrometry (DESI-MS) imaging. J. Agric. Food Chem. 61, 7477-7487 (2013)

22. Manicke, N.E., Kistler, T., Ifa, D.R., Cooks, R.G., Ouyang, Z.: Highthroughput quantitative analysis by desorption electrospray ionization mass spectrometry. J. Am. Soc. Mass Spectrom. 20, 321-325 (2009)

23. Machado, R.M.D., Toledo, M.C.F., Garcia, L.C.: Effect of light and temperature on the formation of glycoalkaloids in potato tubers. Food Control 18, 503-508 (2007)

24. Nema, P.K., Ramayya, N., Duncan, E., Niranjan, K.: Potato glycoalkaloids: formation and strategies for mitigation. J. Sci. Food Agric. 88, 1869-1881 (2008)

25. Friedman, M., Dao, L.: Distribution of glycoalkaloids in potato plants and commercial potato products. J. Anal. Food Chem. 40, 419-423 (1992)

26. Ha, M., Kwak, J.H., Kim, Y., Zee, O.P.: Direct analysis for the distribution of toxic glycoalkaloids in potato tuber tissue using matrix-assisted laser desorption/ionization mass spectrometric imaging. Food Chem. 133, 11551162 (2012)

27. Caldwell, K.A., Grosjean, O.K., Henika, P.A., Friedman, M.: Hepatic ornithine decarboxylase induction by potato glycoalkaloids in rats. Food Chem. Toxicol. 29, 531-535 (1991)

28. Friedman, M., Rayburn, J.R., Bantle, J.A.: Developmental toxicology of potato alkaloids in the frog embryo teratogenesis assay-Xenopus (FETAX). Food Chem. Toxicol. 29, 537-547 (1991)

29. Cahill, M.G., Caprioli, G., Vittori, S., James, K.J.: Elucidation of the mass fragmentation pathways of potato glycoalkaloids and aglycons using Orbitrap mass spectrometry. J. Mass Spectrom. 45, 1019-1025 (2010)

30. Rokka, V.M., Laurila, J., Tauriainen, A., Laakso, I., Larkka, J., Metzler, M., Pietilä, L.: Glycoalkaloid aglycone accumulations associated with infection by Clavibacter michiganensis ssp. sepedonicus in potato species Solanumacaule and Solanumtuberosum and their interspecific somatic hybrids. Plant Cell Rep. 23, 683-691 (2005)

31. Kuć, J.A.: Metabolites accumulating in potato tubers following infection and stress. Teratology 8, 333-338 (1973) 
32. Rayburn, J.R., Bantle, J.A., Friedman, M.: Role of carbohydrate side chains of potato glycoalkaloids in developmental toxicity. J. Agric. Food Chem. 42, 1511-1515 (1994)

33. Osbourn, A.: Saponins and plant defense - a soap story. Trends Plant Sci. 1, 4-9 (1996)

34. Oda, Y., Saito, K., Ohara-Takada, A., Mori, M.: Hydrolysis of the potato glycoalkaloid alpha-chaconine by filamentous fungi. J. Biosci. Bioeng. 94, 321-325 (2002)

35. Kuc, J.: Steroid glycoalkaloids and related compounds as potato quality factors. Am. Potato J. 61, 123-138 (1982)
36. Wiseman, J.M., Ifa, D.R., Zhu, Y.X., Kissinger, C.B., Manicke, N.E., Kissinger, P.T., Cooks, R.G.: Mass spectrometry across the sciences special feature: desorption electrospray ionization mass spectrometry: imaging drugs and metabolites in tissues. Proc. Natl. Acad. Sci. U. S. A. 105, 18120-18125 (2008)

37. Vismeh, R., Waldon, D.J., Teffera, Y., Zhao, Z.Y.: Localization and quantification of drugs in animal tissues by use of desorption electrospray ionization mass spectrometry imaging. Anal. Chem. 84, 5439-5445 (2012)

38. Ellis, S.R., Bruinen, A.L., Heeren, R.M.A.: A critical evaluation of the current state-of-the-art in quantitative imaging mass spectrometry. Anal. Bioanal. Chem. 406, 1275-1289 (2014) 